\section{A new spin on protegrin}

\section{By Kai-Jye Lou, Staff Writer}

Researchers at the University of Zurich and Polyphor Ltd. have developed protein epitope mimetics of the antimicrobial peptide protegrin I that specifically target Pseudomonas aeruginosa via a mechanism of action that is distinct from the membrane-disrupting activity of the parent compound. ${ }^{1}$ The biotech expects its lead mimetic to enter Phase I testing this year to treat $P$. aeruginosa infections.

$P$. aeruginosa, a Gram-negative bacterium, is a common cause of opportunistic nosocomial infections, and treatment usually involves a course of antibiotics. But the incidence of resistant strains is rising, resulting in the need for new treatments. ${ }^{2,3}$

"Pseudomonas infections that have become multidrug resistant are being treated with polymyxin antibiotics like colistin, which were previously considered too toxic," said Robert Hancock, Canada research chair of the Department of Microbiology and Immunology at The University of British Columbia and director of the university's Centre for Microbial Diseases and Immunity Research. "But even there, we are already seeing strains that are resistant."

He added: "Pseudomonas may be an opportunistic pathogen, but it is causing about 160,000 infections in the U.S. annually."

Protegrin I is an antimicrobial protein, originally isolated from pigs, that induces pore formation in microbial membranes. ${ }^{4}$ It has had a checkered clinical past. In 2004, IntraBiotics Pharmaceuticals Inc. (now Ardea Biosciences Inc.) dropped all development of iseganan. The biotech's synthetic version of protegrin I had failed two Phase III trials in oral mucositis, and a Phase II/III trial of the compound to prevent ventilatorassociated pneumonia (VAP) was terminated after an independent datamonitoring committee found higher rates of VAP and mortality in the treatment arm.

Researchers at the University of Zurich and Polyphor now have shown that several of the biotech's protegrin I-derived protein epitope mimetics appear to kill $P$. aeruginosa through functional inhibition of its LPSassembly protein (ost $\mathrm{A}$ lptD; imp), which is involved with incorporating LPS into the outer leaflet of the bacterium's outer membrane. By contrast to protegrin I's membrane-disrupting action, this approach impairs the bacterium's ability to generate its outer membrane in the first place.

Polyphor's compounds are synthetic cyclic peptide-like molecules that mimic the $\beta$-hairpin and $\alpha$-helix motifs in a protein's secondary structure and thus are able to modulate protein-protein interactions.

The Swiss researchers evaluated the mimetics against more than 100 $P$. aeruginosa clinical isolates and found that the minimum inhibitory concentrations with antimicrobial activity against $90 \%$ of the isolates $\left(\mathrm{MIC}_{90}\right.$ ) were 0.13 and $0.25 \mu \mathrm{g} / \mathrm{mL}$ for the two most potent candidates, POL7001 and POL7080, respectively. A majority of the isolates tested were resistant to one or more classes of antibiotics.

In a mouse septicemia model using two $P$. aeruginosa strains, POL7001 and POL7080 reduced mortality with median effective doses in the $0.25-0.55 \mathrm{mg} / \mathrm{kg}$ range. In contrast, median effective doses of the generic aminoglycoside antibiotic gentamicin were 3.1 and $2.9 \mathrm{mg} / \mathrm{kg}$ against the two strains.

The data were published in Science.

Polyphor expects the lead mimetic, POL7080, to enter Phase I testing by the end of next quarter or early third quarter.

"Our lead compound could be useful for treating patients infected with $P$. aeruginosa, including strains that are resistant to other antibiotics," said John Robinson, a corresponding author on the paper and a professor of organic chemistry at the university. He is also a consultant for Polyphor.

"It looks like a new mechanism and a new target, and there are not many such compounds in development, especially for Gram-negative bacteria like Pseudomonas," said Richard Scott, VP of research at PolyMedix Inc. "It's a very interesting approach and I think it will fit in well with the drugs that are already being used."

Scott told SciBX it was pretty clear that Polyphor's compounds have a mechanism distinct from protegrin $\mathrm{I}$.

"The evidence is strong that the antibiotic -John Robinson, University of Zurich activity is mediated through $l p t D$," he said. "To get such potency, I'd think that they would need to have a specific target-I don't think it would be easy to identify a compound with such high potency if it were targeting the membrane directly because there is much more membrane to disrupt than an individual protein target."

PolyMedix also is developing antimicrobial mimetics. PMX-30063, a small molecule mimic of the host defense protein defensin, is in Phase Ib testing to treat drug-resistant and drug-sensitive Staphylococcal infections. The company expects to start a U.S. Phase II study by the end of next quarter.

\section{Getting specific}

According to Robinson, the broad-spectrum activity of protegrin I was traded for increased potency against $P$. aeruginosa during the optimization process. "We've optimized our compound to attack Pseudomonas and by doing so have reduced its activity against other types of bacteria," he said.

Indeed, POL7001 and POL7080 are highly specific for P. aeruginosa. In an antimicrobial activity assay, both mimetics were inactive against other Gram-negative and Gram-positive bacteria. In contrast, earlier members 


\section{ANALYSIS}

of the series had activity against other types of bacteria, and one of the earliest members showed broad-spectrum activity similar to protegrin I.

Because POL7080 was inactive against other types of bacteria, Polyphor CFO Michael Altorfer thinks using it to treat $P$. aeruginosa will not run the risk of resistance-promoting selective pressure in off-target bacteria.

"Treatment with broad-spectrum antibiotics is known to promote antibiotic resistance because these drugs can have weak activity against some of the other bacterial strains that are present," he said. "This could promote the development of resistance in off-target strains."

Scott was more cautious, noting that bacteria may have an easier time evolving resistance to a compound that hits a single target like lptD rather than "a more general mechanism like membrane disruption."

Altorfer countered that "the resistance frequency for POL7080 is in the same range with that of other antibiotics, if not lower."

Robinson added that $P$. aeruginosa strains that became resistant to POL7080 did not also become resistant to other classes of antibiotics, including colistin, which kills bacteria by disrupting their cytoplasmic membranes. He also noted that Pseudomonas strains that became resistant to POL7080 "appeared to become more sensitive to other antibiotics like ciprofloxacin. This observation is something that we want to follow up with."

Ciprofloxacin is a fluoroquinolone antibiotic.

Robinson said his group is still working to pin down the details of the mechanism of action of their mimetics. "We also want to go back along the structure-activity trail and develop compounds with broader-spectrum activity against other types of Gram-negative bacteria," he said.

Polyphor and the university have jointly filed multiple patent applications covering protein epitope mimetics with antibiotic and antimicrobial activity. POL7080 and the follow-on research program to assess the antibacterial activity of other mimetics against other types of Gramnegative bacteria are available for licensing from the company.

Lou, K.-J. SciBX 3(9); doi:10.1038/scibx.2010.265

Published online March 4, 2010

\section{REFERENCES}

1. Srinivas, N. et al. Science; published online Feb. 18, 2010; doi:10.1126/science.1182749

Contact: John A. Robinson, University of Zurich, Zurich, Switzerland e-mail: robinson@oci.uzh.ch

Contact: Steven J. DeMarco, Polyphor Ltd., Allschwil, Switzerland e-mail: steve.demarco@polyphor.com

2. Poole, K. Antimicrob. Agents Chemother. 49, 479-487 (2005)

3. Merlo, C.A. et al. Chest 132, 562-568 (2007)

4. Bolintineanu, D. et al. Peptides 31, 1-8 (2010)

\section{COMPANIES AND INSTITUTIONS MENTIONED}

Ardea Biosciences Inc. (NASDAQ:RDEA), San Diego, Calif.

PolyMedix Inc. (OTCBB:PYMX), Radnor, Pa.

Polyphor Ltd., Allschwil, Switzerland

The University of British Columbia, Vancouver, British Columbia, Canada University of Zurich, Zurich, Switzerland 